\title{
Hommage au Professeur G. De Mello et à l'ami Gilbert
}

Tu étais un noble épicurien mais aussi un homme de devoir et de conviction qui a toujours œuvré pour la promotion de la Médecine bucco-dentaire et plus particulièrement celle de la Chirurgie buccale, sans jamais oublier celle de la Médecine buccale. Tu as exercé de nombreuses responsabilités :

- PU-PH depuis 1998, Chef du Service de Chirurgie et de Pathologie buccales et Adjoint au chef de pôle au CHU de Rennes;

- Doyen de l'UFR d'Odontologie de Rennes depuis 2008;

- Membre du CNU ;

- Expert près du Conseil scientifique de l'Internat;

- Président de la Société Francophone de Médecine buccale et Chirurgie buccale (2000-2004); pendant tes deux mandats, tu as pris des initiatives (rédaction de Recommandations, développement de la revue Médecine buccale Chirurgie buccale...) qui ont donné un nouvel élan à la SFMBCB ;

- Membre du Comité de rédaction de la revue Médecine buccale Chirurgie buccale depuis le $1^{\text {er }}$ numéro paru en septembre 1995 jusqu'au $4^{\text {ème }}$ numéro de l'année 2009 ;

- Trésorier de la CID-CDF (Conférence internationale des doyens et des facultés de Chirurgie dentaire d'expression totalement ou partiellement francophone).

Tu as permis à de nombreux confrères d'obtenir le DESCB et d'acquérir une solide formation qui fait de la Bretagne probablement la région de France où il y a le plus de praticiens libéraux exclusifs en Chirurgie buccale. Pour toi la formation clinique devait être associée à une recherche clinique constante afin d'améliorer sans cesse la prise en charge des patients. Depuis quelques années, tu t'es surtout investi pour faire évoluer l'approche des troubles de l'hémostase (congénitaux ou acquis) en Médecine bucco-dentaire et tu as réussi à ouvrir une brèche dans l'empirisme et l'archaïsme qui prévalaient dans ce domaine (cf. tes articles «Étude épidémiologique sur les extractions dentaires chez les patients sous AVK en Ille-et-Vilaine » et « Étude du rapport coût/sécurité lors de la prise en charge des patients sous antivitamines $\mathrm{K}$ en chirurgie buccale », parus dans le $n^{\circ} 1 / 2009$ de la revue Médecine Buccale et Chirurgie Buccale). Dernièrement, toujours poussé par ta curiosité instinctive et ta polyphagie intellectuelle, tu as relevé un nouveau défi, en t'aventurant dans le domaine de la recherche fondamentale (cf. ton article dans ce numéro sur la « Mise au point d'un modèle tridimensionnel pour l'évaluation des biosubstituts osseux in vitro »). Dans toutes tes activités et dans toutes les actions que tu as mené, tu as fais preuve d'un enthousiasme communicatif et d'une approche rigoureuse mais toujours conviviale car tu étais, sans doute comme Montesquieu, convaincu que « La gravité est le bonheur des imbéciles». Mais tu étais trop affable pour t'en tenir à cette expression péremptoire, tu étais incontestablement plus proche de Christophe, pour qui les « Les gens sérieux ne sont jamais graves... et les gens graves ne sont jamais sérieux ». En réalité, sous ton apparence de sybarite, se cachait un adepte éclairé du stakhanovisme, considérant que l'exemple donné constitue la meilleure base de l'émulation.

Tu avais l'apparence d'un être simple et tu avançais dans la vie sans fard et sans détour. La générosité de ton abdomen faisait suspecter un féroce appétit mais, en réalité, tu étais un gourmet averti et non un vil gourmand. Tu aimais partager le plaisir de la table dans une sorte de communion païenne et faire découvrir ce que tu appréciais. Tu avais toujours un mot pour nous éclairer sur la charcuterie, les fruits de mer, la viande, les abats, les champignons, les fromages y compris les meilleurs fromages anglais... et, sur le vin, tu étais intarissable. Certes tu aimais déguster des grands crus mais il y a bien longtemps que tu avais saisi que ce n'est pas la qualité qui détermine le prix. Aucune carte des vins n'avait de secret pour toi et tu avais l'art de dénicher un bon produit à un prix raisonnable, tout en nous faisant participer à ta démarche diagnostique. Tu accompagnais toujours le choix d'un vin de digressions qui nous donnaient un instant l'illusion d'avoir bénéficié d'un transvasement culturel mais, après décantation, il nous en restait souvent peu de choses. Avec le temps, tu avais néanmoins réussi à nous inculquer quelques rudiments d'œnologie. Tu avais également investigué les produits dérivés du vin, toujours en quête d'autres sensations olfactives et gustatives. Manifestement, tu préférais le cognac à l'armagnac et, quand tu as découvert le Louis XIII, tu es resté un bon moment sans parler, déglutissant encore et encore la première gorgée pour en exhaler toutes les saveurs, et tes yeux écarquillés étaient figés par le plaisir. 
A table, comme dans la vie, tu n'avais pas besoin de parler pour t'exprimer. Tes petits yeux, pleins de malice, reflétaient instantanément ta satisfaction, ton acquiescement, ton étonnement, ton incrédulité, ton courroux... Tu avais le rire facile mais discret et toujours gai. Assez souvent, tu esquissais un demi-sourire volontiers dubitatif ou légèrement sarcastique, à peine perceptible sous ton épaisse moustache. Dans ton regard et dans ton sourire, comme dans tes paroles d'ailleurs, il n'y avait jamais de haine ni de mépris. Tu étais un homme droit et généreux et, malgré ton exil en Bretagne, tu avais gardé un gros cœur comme beaucoup de gens du sud et ta tête était encore pleine du soleil de ton enfance méditerranéenne.
Gilbert, nous parlerons encore longtemps de toi et, chaque fois que nous commanderons des Gillardeau $n^{\circ} 3$ et une bouteille de Quincy, nous aurons un petit pincement précordial et au moins une déglutition à vide pour refréner notre émotion.

Pour Annette, ta moitié anthropomorphique, et pour tes enfants, nous avons une pensée émue et nous compatissons à l'affliction provoquée par ta disparition inopinée. 